\title{
Spontaneous Marangoni Mixing of Miscible Liquids at a Liquid- Liquid-Air Contact Line
}

\author{
Hyoungsoo Kim, ${ }^{*}{ }^{\dagger}$ Jeongsu Lee, ${ }^{*}$ Tae-Hong Kim, ${ }^{\ddagger}$ and Ho-Young Kim* ${ }^{*}$ \\ ${ }^{\dagger}$ Department of Mechanical and Aerospace Engineering, Princeton University, Princeton, New Jersey 08544, United States \\ ${ }^{\ddagger}$ Department of Mechanical and Aerospace Engineering, Seoul National University, Seoul 151-744, Republic of Korea
}

\section{Supporting Information}

ABSTRACT: We investigate the flow patterns created when a liquid drop contacts a reservoir liquid, which has implications on various physicochemical and biochemical reactions including mixing in microfluidic systems. The localized vortical flow spontaneously triggered by the difference of surface tension between the two liquids is studied, which is thus termed the Marangoni vortex. To quantitatively investigate the strength of vortices, we performed particle image velocimetry (PIV) experiments by varying the surface tension difference, the gap of the flow cell, the density and viscosity of the reservoir liquid, and the size of the drop. A scaling law that balances the interfacial energy of the system with the kinetic energy of the vortical flows allows us to understand the functional dependence of the Marangoni vortex strength on various experimental parameters.

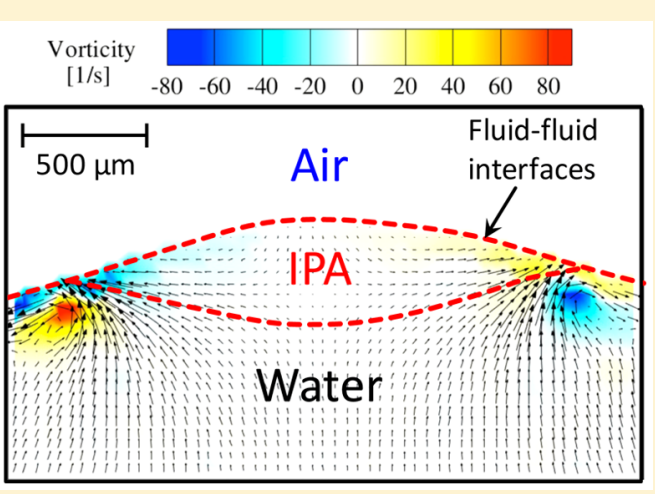

\section{INTRODUCTION}

Mixing of different liquids confined within a small volume is an important issue in many emerging technologies, such as lab-ona-chip, ${ }^{1}$ semiconductor cleaning, ${ }^{2,3}$ and polymer processing. ${ }^{4}$ In a microfluidic system where two channels filled with different liquids merge at a junction to form a single channel, molecular diffusion is mainly responsible for mixing of the two liquids unless some mixing scheme is devised. Active mixers use external energies (e.g., mechanical, electrical, and magnetic effect) to resonate the mixing, ${ }^{1,5,6}$ while passive mixing enhances the mixing efficiency by using a complex geometry. ${ }^{7,8}$ However, when the liquids in contact are exposed to another fluid, such as air, the mixing flow characteristics at the interface can be changed qualitatively, as shown in Figure 1. While isopropyl alcohol (IPA) and water mix only through molecular diffusion in Figure 1a, rapid swirls are spontaneously generated when IPA locally touches the free surface of water, as shown in Figure $1 \mathrm{~b}$. The rotating flows generated at the water-IPA-air contact line can enhance mixing efficiency without any additional device.

The circulating flow pattern that arises when a liquid partially covers the free surface of the other reservoir liquid was first reported several decades ago. ${ }^{9}$ It was aptly attributed to the Marangoni effect that generates flows at a fluid-fluid interface due to surface tension gradient. Formation of the liquidliquid-air contact line is a typical process involved in either the coalescence of a liquid drop with the other liquid or the propagation of a liquid film on top of the other liquid, which has been a subject of intense study. ${ }^{10-21}$ The previous studies were, however, mainly focused on the shape evolution of the drop or film. Hence, quantitative understanding of the rotating flows near the liquid-liquid-air contact line is far from

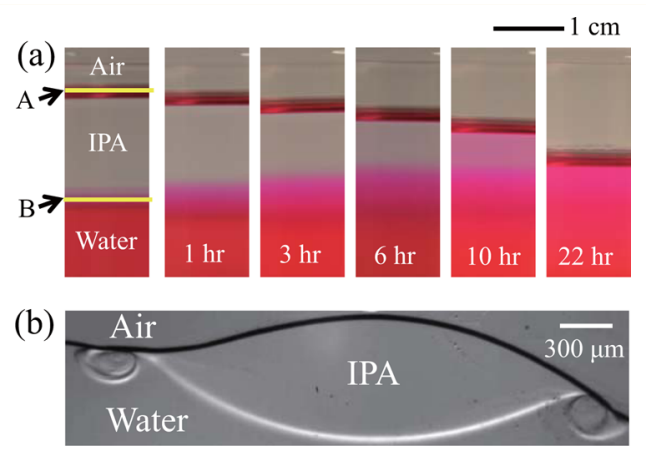

Figure 1. Mixing of IPA and water. (a) Diffusive mixing at the IPAwater interface. Water is initially colored in pink with a red food dye (Innovating Science). Lines A and B indicate the air-IPA interface and the IPA-water interface, respectively. The level of IPA falls with time due to evaporation, while the IPA and water are mixed by diffusion. (b) Swirls generated upon the deposition of an IPA drop on the water-air interface in a flow cell with $1 \mathrm{~mm}$ gap. The depth of water in the vertical direction is $\sim 2 \mathrm{~cm}$.

complete. In the following, we first investigate the vortical flow structures resulting from the Marangoni effect by using PIV measurement technique. We then give a simple theory to correlate the magnitude of circulation with various experimental parameters including the dominant cause of the process, the surface tension difference of the two liquids.

Received: May 22, 2015

Revised: July 13, 2015

Published: July 17, 2015 


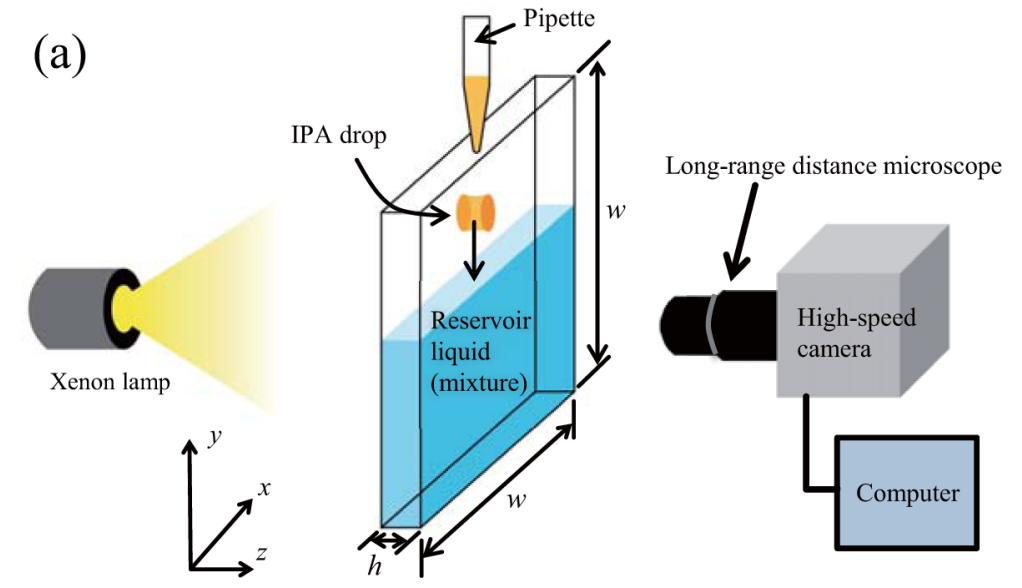

(b)

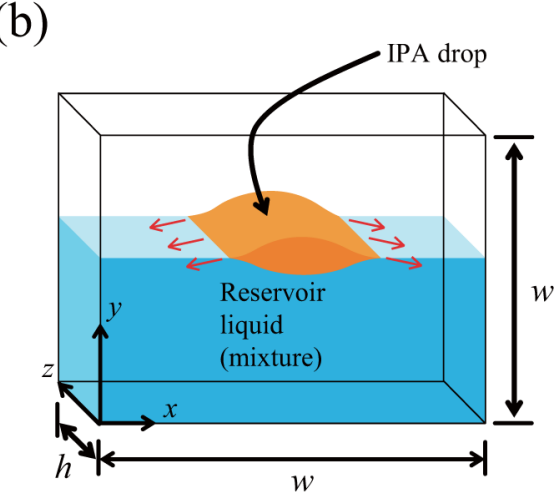

Figure 2. (a) Experimental apparatus. The reservoir liquid is confined within a vertical flow cell with the gap $h=160$ or $240 \mu \mathrm{m}$ and the side length $w=24 \mathrm{~mm}$. The depth of reservoir liquid is $\sim 2 \mathrm{~cm}$. (b) Schematic of a floating IPA drop on the reservoir liquid where red arrows represent the direction of Marangoni force, which is parallel to the cell walls.

\section{EXPERIMENTAL SECTION}

We prepare an experimental setup to visualize vortices induced by the Marangoni effect, as shown in Figure 2. A drop of IPA (99.5\%, SigmaAldrich) emitted from a pipet slides down through the gap of two parallel cover glasses (Deckglaser, Germany) to touch the reservoir liquid. IPA has the dynamic viscosity $\mu=2.16 \mathrm{cP}$, the density $\rho_{1}=786$ $\mathrm{kg} / \mathrm{m}^{3}$, and the surface tension $\sigma_{1}=20.2 \mathrm{mN} / \mathrm{m}$. The drop volume $V$ ranges from 100 to $400 \mathrm{~nL}$, so that the equivalent diameter of the shallow cylinder ranges from 490 to $915 \mu \mathrm{m}$. As the reservoir liquids, we use mixtures of deionized water (Human Ro 180) and ethanol (200 proof, anhydrous, 99.5\%, Sigma-Aldrich) with different volume fractions, whose properties are listed in Table 1. The cover glasses

Table 1. Physical Properties of Water-Ethanol Mixtures at $T$ $=295 \mathrm{~K}$ Based on Literature Values ${ }^{22,23}$

$\begin{array}{ccccc}\begin{array}{c}\text { mixture } \\ \text { no. }\end{array} & \begin{array}{c}\text { ethanol volume } \\ \text { fraction }(\%)\end{array} & \begin{array}{c}\text { density } \\ \left(\mathrm{kg} / \mathrm{m}^{3}\right)\end{array} & \begin{array}{c}\text { surface tension } \\ (\mathrm{mN} / \mathrm{m})\end{array} & \begin{array}{c}\text { viscosity } \\ (\mathrm{cP})\end{array} \\ 0 & 0 & 1000 & 72.7 & 1.0 \\ 1 & 20 & 979 & 41.5 & 1.48 \\ 2 & 40 & 953 & 32.3 & 2.07 \\ 3 & 50 & 937 & 29.8 & 2.32 \\ 4 & 60 & 917 & 27.8 & 2.44 \\ 5 & 80 & 869 & 24.8 & 2.07\end{array}$

constituting the vertical flow cell are cleaned in an acetone solution in the ultrasonic bath for $5 \mathrm{~min}$, rinsed with deionized water, and dried using nitrogen gas before use. The contact angle of water and IPA with a cover glass is measured to be 55 and $10^{\circ}$, respectively. The contact angles of ethanol-water mixtures with a cover glass are 54 (ethanol 5 wt \%), 52 (ethanol $10 \mathrm{wt} \%$ ), 38 (ethanol $30 \mathrm{wt} \%$ ), 26 (ethanol $50 \mathrm{wt}$ $\%$ ), 25 (ethanol $70 \mathrm{wt} \%$ ), and $15^{\circ}$ (ethanol $90 \mathrm{wt} \%$ ), with the typical standard deviation being $3^{\circ}$. The contact angles are determined from a linear fit to the liquid interface profile within a distance of $50 \mu \mathrm{m}$ from the corner of each drop sitting on the horizontally situated cover glass. Two different separations of the glass walls are employed in constructing the flow cells: 160 and $240 \mu \mathrm{m}$. The gap thickness is varied with a double-sided film tape (3M, USA), and is measured by a micrometer. The edges of the channel are sealed with melted polyethylene. The cell position is controlled with a linear stage (Newport, USA) for flow visualization.

To visualize the fluids motion, we seed hydrophilic polystyrene polymer particles of $19 \mu \mathrm{m}$ diameter (Duke Scientific) in waterethanol mixtures and dried hydrophobic polyamide particles of $20 \mu \mathrm{m}$ diameter (Dantec Dynamics) in IPA. The Stokes number, defined as $\mathrm{St}=\tau_{p} / \tau_{l}$, where $\tau_{p}$ is the particle response time and $\tau_{l}$ is the time scale of fluid motion, is on the order of $10^{-4}$ because $\tau_{p}\left(=(1 / 18) \rho_{p} d_{p}^{2} / \mu_{l}\right)$ $\approx 10^{-5} \mathrm{~s}$ and $\tau_{l}(=a / U) \approx 10^{-1} \mathrm{~s}$, where $\rho_{p}$ is the density of the particle $\left(1050 \mathrm{~kg} / \mathrm{m}^{3}\right.$ for polystyrene and $1030 \mathrm{~kg} / \mathrm{m}^{3}$ for polyamide particles), $d_{p}$ is the diameter of the particle, $a$ is the vortex radius, and $\mu_{l}$ is the mixture viscosity. ${ }^{24} U$ is the flow speed, $O(1 \mathrm{~mm} / \mathrm{s})$, as obtained from the PIV measurement. Therefore, the particles can be
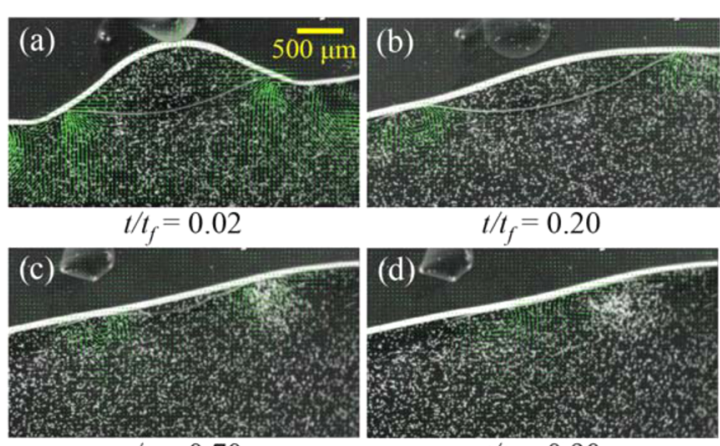

$t / t_{f}=0.70$

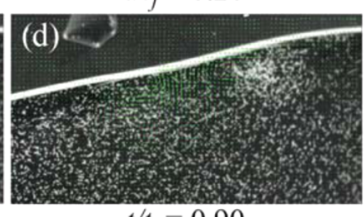

$t / t_{f}=0.90$

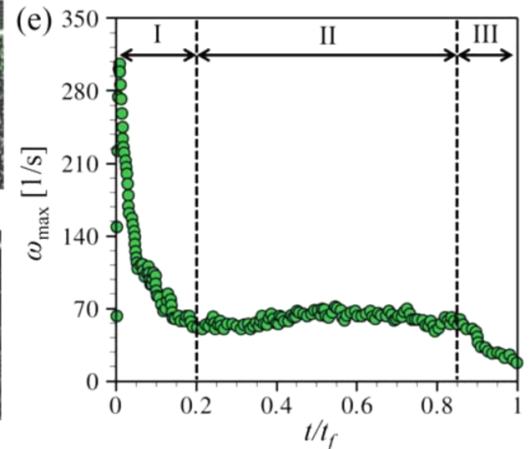

Figure 3. $(a-d)$ Temporal evolution of the shape of an IPA drop and flow fields as the IPA drop is deposited on the water (80\%)-ethanol (20\%) mixture by volume. Images are captured from the supplementary movie in the Supporting Information. The green vectors are obtained by PIV measurement. (e) Maximum vorticity, $\omega_{\max }$ versus $t / t_{f}$, where the total mixing time $t_{f}$ is $2.75 \mathrm{~s}$. (I) initial drop impact and spreading, (II) steady vortex generation, and (III) decaying period. 


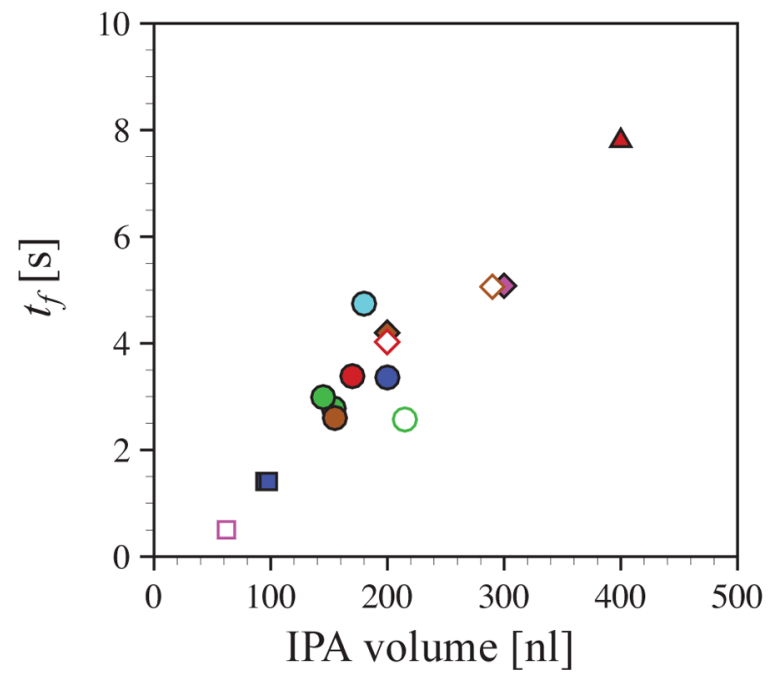

\begin{tabular}{|c|c|c|c|}
\hline Symbol & $\Delta \sigma[\mathrm{mN} / \mathrm{m}]$ & $V[\mathrm{n}]$ & $h[\mu \mathrm{m}]$ \\
\hline \hline$\triangle$ & 51 & 400 & 160 \\
\hline$\bigcirc$ & 51 & 170 & 160 \\
\hline$\bigcirc$ & 20 & 145,154 & 160 \\
\hline$\square$ & 11 & 95,98 & 160 \\
\hline$\bigcirc$ & 11 & 200 & 160 \\
\hline$\bigcirc$ & 8 & 180 & 160 \\
\hline$\bigcirc$ & 6 & 300 & 160 \\
\hline$\square$ & 6 & 66 & 160 \\
\hline$\bigcirc$ & 3 & 155 & 160 \\
\hline$\bigcirc$ & 3 & 201 & 160 \\
\hline$\bigcirc$ & 51 & 215 & 240 \\
\hline$\bigcirc$ & 20 & 193 & 240 \\
\hline$\square$ & 6 & 62 & 240 \\
\hline$\searrow$ & 3 & 290 & 240 \\
\hline
\end{tabular}

Figure 4. Total mixing time, $t_{f}$ versus initial IPA drop volume for various experimental conditions. The total mixing rate is on the order of $50 \mathrm{~nL} / \mathrm{s}$.
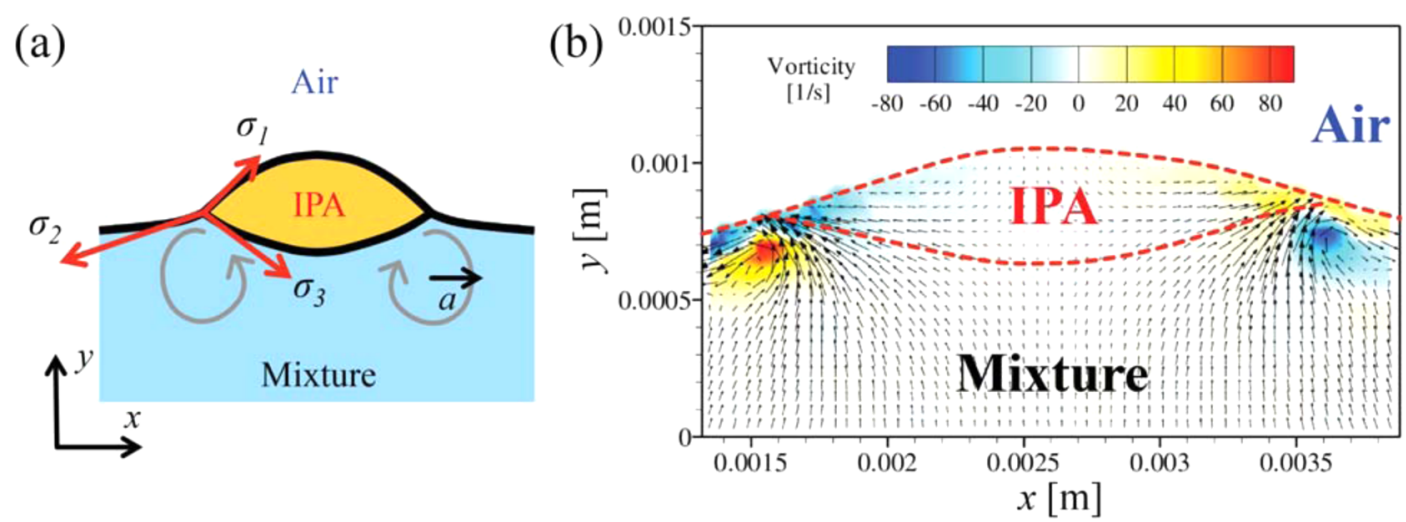

Figure 5. (a) Schematic of surface tension forces (red arrows) and vortices (gray arrows) at the liquid-liquid-air contact line. $\sigma_{1}, \sigma_{2}$, and $\sigma_{3}$ denote the interfacial tension between IPA and air, water-ethanol mixture and air, and IPA and water-ethanol mixture, respectively. $a$ is the radius of vortex. The vortex size is defined by setting a threshold value of $35 \%$ of the maximum vorticity. The radius is calculated as an equivalent radius of the area. (b) 2D-PIV result for an IPA drop with the initial volume of $150 \mathrm{~nL}$ floating on the $20 \%$ ethanol-water mixture (mixture no. 1 in Table 1 ). The red dashed lines indicate the interfaces, that is, the air-IPA interface, air-mixture interface, and IPA-mixture interface. The black arrows represent $2 \mathrm{D}$ velocity vectors and the color contours indicate vorticity magnitudes.

assumed to follow the fluid motion almost perfectly. A xenon HID (high intensity discharge) lamp (Polarion) is used as a light source. Directly counting particles from the raw images yields a value of $\sim 0.01$ ppp (particles per pixel). The particle movements are captured at the frame rate of $500 \mathrm{~s}^{-1}$ by a high-speed CMOS (complementary metaloxide semiconductor) camera (Photron SA 1.1) having a pixel resolution of $1376 \times 1040$ and a 12-bit dynamic range. We use a longrange microscope lens (Navitar). Under this experimental condition, the depth of field of the PIV setup covers the whole channel gaps $(h=$ 160 and $240 \mu \mathrm{m})$. All particles are uniformly distributed in the channel, and we recorded the movement of the projected particles that contribute to the cross-correlation. The measured diameter of the particle image is $\sim 5$ pixels. During the measurement, the room temperature is kept at $295 \pm 2 \mathrm{~K}$, which is measured next to the vertical flow cell.

To calculate the velocity vectors, the planar cross-correlation is applied with a chosen interrogation window of $24 \times 24$ pixels at $50 \%$ overlap. In this PIV calculation, the location of the peak of the correlation map is typically ranged from 5 to 10 pixels. Therefore, the most probable velocity displacement of the particles is relevant to the current interrogation window size. Furthermore, the random error of the PIV measurement is $\sim 0.05$ pixel units for a given interrogation domain. ${ }^{24}$ To reliably detect the particles, we use a high-pass filtering to improve the sharpness of the blurred particle images and a $5 \times 5$ Gaussian smoothing to reduce noise of the particle images. ${ }^{24}$ Spurious velocity vectors may occur due to a background noise outside the measurement domain and the fluids interface during the crosscorrelation. Hence, we remove these outliers by applying a mask filter based on the measurement images. ${ }^{25}$ The whole pre- and postprocessing procedures are performed by using Matlab.

\section{RESULTS AND DISCUSSION}

The flow-field measurements are performed by using PIV. Figure $3 \mathrm{a}-\mathrm{d}$ displays the typical flow characteristics arising from the contact of a liquid with the free surface of the other liquid. (See the detailed flow pattern in the supplementary movie in the Supporting Information.) In this case, the IPA drop $(V \approx 150 \mathrm{nl})$ is deposited on the $20 \%$ ethanol-water mixture (mixture no. 1 in Table 1). The interface between the floating IPA drop and the reservoir liquid is nearly perpendicular to the cell walls, thereby yielding the liquidliquid-air contact line parallel to the $z$ axis. Furthermore, the Marangoni force points in a direction parallel to the side wall, as schematically shown in Figure $2 b$, allowing us to assume that the velocity vectors are almost parallel to the cell walls with negligible $z$-directional components. We show the temporal evolution of vorticity in Figure $3 \mathrm{e}$, where $\omega_{\max }$ is the maximum vorticity located at the vortex core. The flow evolves through 

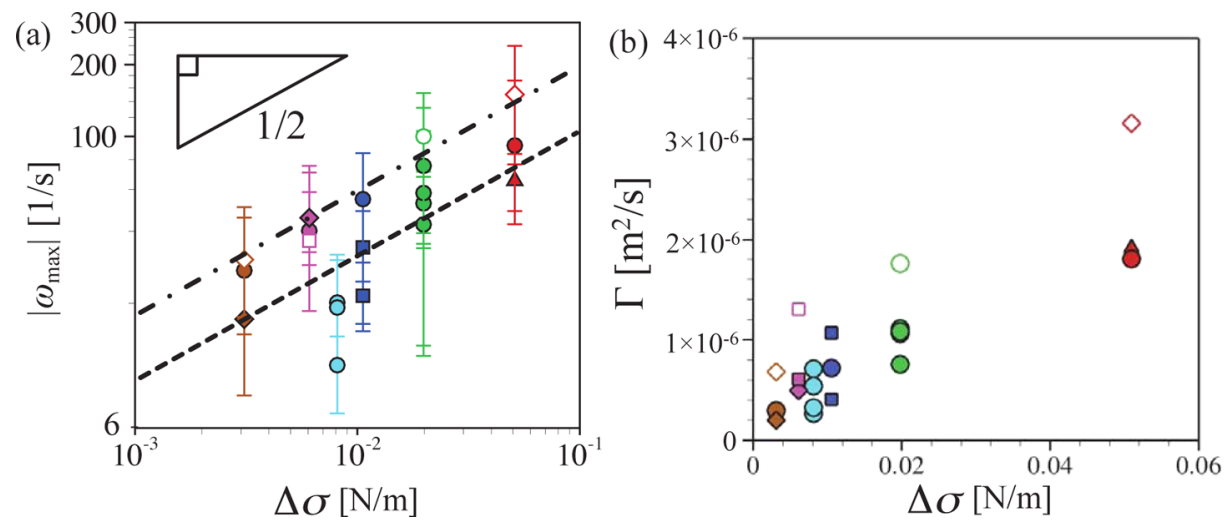

Figure 6. Effects of surface tension difference $\Delta \sigma=\sigma_{2}-\sigma_{1}$ on (a) maximum vorticity $\omega_{\max }$ and (b) vortex strength $\Gamma$. The experimental conditions for each symbol are identical to those in Figure 4. The dashed line and filled symbols correspond to the channel gap of $160 \mu \mathrm{m}$, and the dasheddotted line and empty symbols correspond to the gap of $240 \mu \mathrm{m}$. In panel a, the fitting lines correspond to $\omega_{\max } \approx \Delta \sigma^{1 / 2}$.

the following three stages: (I) initial drop impact and spreading $\left(t / t_{f}=0\right.$ to 0.2$)$, (II) steady vortex generation at the contact line $\left(t / t_{f}=0.2\right.$ to 0.85 ), and (III) decay of vortex (after $t / t_{f}=$ $0.85)$, where $t_{f}$ is the total mixing time, $2.75 \mathrm{~s}$ in this case. The Marangoni vortex continues to be generated while the IPA drop spreads along the water-ethanol mixture surface that is exposed to air. As the IPA drop shrinks due to mixing, the two vortices near the liquid-liquid-air contact lines get closer. Eventually they collide when the drop vanishes; this instant is noted as $t_{f}$. The total mixing time $t_{f}$ including regimes I, II, and III is proportional to the initial IPA volume, as shown in Figure 4. The slope of Figure 4 allows us to estimate the total mixing rate, which is on the order of $50 \mathrm{~nL} / \mathrm{s}$.

We schematically illustrate the forces and flows occurring when an IPA drop sits on a water-ethanol mixture surface in Figure 5a. Because IPA has a lower surface tension than that of water-ethanol mixtures, the liquid in the drop is drawn onto the reservoir liquid and then swirls, inducing vigorous mixing of the two liquids. (See the supplementary movie in the Supporting Information.) Figure $5 \mathrm{~b}$ shows a $2 \mathrm{D}$ velocity field obtained by PIV in the steady vortex generation stage. The drop liquid is drawn tangent to the interface of water-ethanol mixture and air at the contact line. Such liquid motion in the mixture brings about the rise of the liquid beneath the contact line. Then, the tangentially outward flows near the contact line rotate to satisfy the mass conservation. Because the drop liquid is continually drawn onto the reservoir, the vortices are generated steadily until the drop vanishes.

To quantify the strength of flow rotation generated at the contact line, we evaluate the circulation $\Gamma=\int_{A} \omega \mathrm{d} A$, where $\omega$ is the vorticity and $A$ is the area of vortex defined by setting a threshold value of $35 \%$ of the maximum vorticity. We calculate the vortex strength during the steady vortex generation period. (See regime II of Figure 3e.) While this threshold value is rather arbitrary, the results trend does not change significantly with different threshold values. ${ }^{26}$ For each experimental condition, we take the arithmetic average of $\Gamma$ over the steady vortex generation duration. Figure 6 shows the effects of the surface tension difference, $\Delta \sigma=\sigma_{2}-\sigma_{1}$, and channel gap, $h$, on the vorticity magnitude and the circulation. We see that $\omega_{\max }$ and $\Gamma$ increase with the increase in $\Delta \sigma$ and $h$. Furthermore, we obtain $\omega_{\max } \approx \Delta \sigma^{1 / 2}$, as shown in Figure 6a. To further understand this functional dependence, we construct a simple theoretical model for the circulation of the Marangoni vortices in the following.
We start with dimensional analysis for regime II where the vortices are generated steadily. Our experimental observations and physical considerations lead us to assume that the vortex strength per depth $\Gamma / h$ must be a function of the surface tension difference $(\Delta \sigma)$, the density of the rotating fluid (i.e., the reservoir liquid) $\left(\rho_{2}\right)$, the deposited drop size $(R)$, and the deposited drop's liquid-air surface tension $\left(\sigma_{1}\right)$. Therefore, we write $\Gamma / h=f\left(\Delta \sigma, \rho_{2}, R, \sigma_{1}\right)$. According to the Buckingham $\Pi$ theorem, ${ }^{27}$ we obtain the following dimensionless relationship

$$
\frac{\Gamma / h}{\left[\Delta \sigma /\left(\rho_{2} R\right)\right]^{1 / 2}}=f\left(\frac{\sigma_{1}}{\Delta \sigma}\right)
$$

In the following, we use physical arguments to determine the functional dependency of the scaled parameters and experimentally examine the results.

After an IPA drop is deposited on a reservoir liquid, the liquids are mixed to run down to equilibrium, which is manifested by vortices. Three physical mechanisms can be at work, minimization of interfacial energy, gravity, and molecular diffusion. The Bond number defined as Bo $=\Delta \rho g R^{2} / \Delta \sigma$, with $\Delta \rho$ being the density difference of IPA and water-ethanol mixture and $g$ being the gravitational acceleration, ranges from $2 \times 10^{-3}$ to $4 \times 10^{-1}$. Therefore, the gravitational effect is relatively negligible compared with the interfacial effects. It is clear that molecular diffusion is insignificant in view of vigorously stirring vortical flows, which renders the Peclet number, $\mathrm{Pe}=U h / D \approx 10^{3}$. Here we take $D$ as the typical diffusion coefficient of IPA in water $\left(1.03 \times 10^{-9} \mathrm{~m}^{2} / \mathrm{s}\right)$. Therefore, the force due to surface tension gradient is a dominant driving force responsible for mixing of the two liquids.

The kinetic energy of the vortical flow, $E_{k}$, is scaled as $E_{\mathrm{k}} \approx$ $\left(\rho_{2} a^{2} h\right)(a \omega)^{2}$, where $\rho_{2}$ is the density of the water-ethanol mixture and $a$ and $\omega$ are the radius and the mean vorticity of the vortex, respectively. Thus, we write $E_{k} \approx \rho_{2} \Gamma^{2} h$ for $\Gamma \approx \omega a^{2}$. The capillary potential energy is converted into the kinetic energy of the vortical flow, which can be written as

$$
\rho_{2} \Gamma^{2} h \approx \Delta \sigma h U \tau
$$

The Marangoni convective flow speed $U$ is scaled as $U \approx$ $\left[\Delta \sigma h /\left(\rho_{1} R^{2}\right)\right]^{1 / 2}$, which is obtained by balancing the capillary force $\Delta \sigma h$ with the inertial force of the IPA drop $\rho_{1} U^{2} \mathrm{R}^{2}$. The mixing time is thus scaled as $\tau \approx\left(\rho_{1} h^{3} / \sigma_{1}\right)^{1 / 2}$. ${ }^{16}$ Here we have neglected the viscous effects as compared with the inertial 
effects by considering the ratio of the viscous time scale taken to be $\tau_{v}=\mu h / \sigma_{1}$ to the inertial time scale $\tau: \tau_{v} / \tau$. It corresponds to the Ohnesorge number, $\mathrm{Oh}=\mu /\left(\rho_{1} h \sigma_{1}\right)^{1 / 2}$, which ranges from $8 \times 10^{-3}$ to $2 \times 10^{-2}$. We note that $\mathrm{Oh}$ is analogous to the inverse of the Reynolds number if a characteristic velocity is defined as $U=h / \tau \approx\left(\sigma_{1} / \rho_{1} h\right)^{1 / 2}$. The Reynolds number $\operatorname{Re}=$ $\rho_{1} \omega R^{2} / \mu$, defined based on the drop size and measured velocity of rotating flow, ranges from 60 to 200 . Then, we get

$$
\rho_{2} \Gamma^{2} h \approx \Delta \sigma h\left(\frac{\Delta \sigma h}{\rho_{1} R^{2}}\right)^{1 / 2}\left(\frac{\rho_{1} h^{3}}{\sigma_{1}}\right)^{1 / 2}
$$

This finally yields

$$
\Gamma \approx\left(\frac{h^{2} \Delta \sigma^{3 / 2}}{\rho_{2} R \sigma_{1}^{1 / 2}}\right)^{1 / 2}
$$

We plot the experimental measurement results according to the scaling law in Figure 7, which shows that the scattered data in

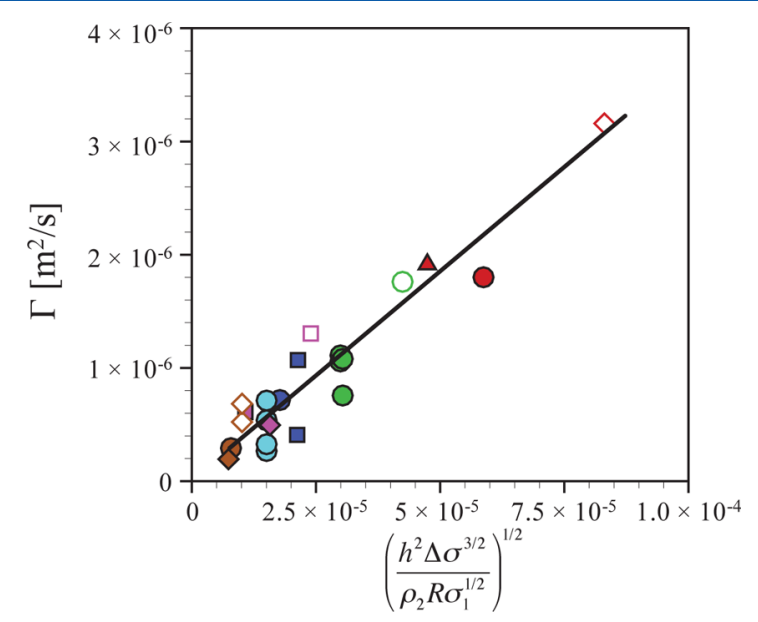

Figure 7. $\Gamma$ plotted according to the scaling law 4 . The experimental conditions for each symbol are identical to those in Figure 4. The slope of the best fitting straight line is 0.036 .

Figure 6 tend to gather closely on a straight line. The scatters of the data remaining in Figure 7 are consequences of the simplification made in the scaling analysis and limits in experimental measurements, which lead us to think about future sophistication of our model and experiment. In particular, considering the variation of the flow velocity in the thickness direction of the cell and the effects of viscosity is anticipated to improve the accuracy of the theoretical predictions. Also, the threshold value of the vortex detection affects the final values of circulation, although the overall trend observed in Figures 6 and 7 is invariant.

Before we conclude, we note that the scaling law 4 is consistent with not only our experimental observations but also the dimensional analysis result. Rearranging scaling law 4 gives

$$
\frac{\Gamma}{h} \approx\left(\frac{\Delta \sigma}{\rho_{2} R}\right)^{1 / 2}\left(\frac{\sigma_{1}}{\Delta \sigma}\right)^{-1 / 4}
$$

which is in accordance with eq 1 .

\section{CONCLUSIONS}

In summary, we have visualized and quantified rapidly swirling flows originated from the contact line of miscible liquids with air using PIV. The flow is driven by the Marangoni effect that arises when the two liquids having a different surface tension meet. Balancing the capillary (potential) energy of the system with the kinetic energy of the rotating flow allows us to explain the functional dependency of the vortex strength or circulation on the surface tension, drop size, and flow cell size.

While we have neglected the effects of viscosity in view of small $\mathrm{Oh}$, the scaling analysis needs to be modified if $\mathrm{Oh}$ increases. If the Marangoni force is mainly balanced by the viscous force, we get $\mu U R^{2} / h \approx \Delta \sigma h$, which gives the Marangoni convective velocity $U \approx \Delta \sigma h^{2} /\left(\mu R^{2}\right)$. Then, it follows that the vortex strength decreases with the increase in viscosity. The detailed analysis of the dependency of vortex strength and circulation upon increased viscosity and reduced drop and gap size is worthy of further study. It is likely to involve numerical computations, which fall beyond the scope of this work that mainly focuses on elucidating a dominant physical mechanism underlying the novel experimental flow phenomena. The rapid mixing at the liquid-liquid interface exposed to air as observed in this work can be used for efficient mixers in lab-on-a-chip systems, ${ }^{28}$ where our simple theoretical framework can guide the optimal design of microscale flow networks.

\section{ASSOCIATED CONTENT}

\section{S Supporting Information}

Video of the temporal evolution of the shape of an IPA drop and flow fields as the IPA drop is deposited on the water (80\%)-ethanol (20\%) mixture by volume. The Supporting Information is available free of charge on the ACS Publications website at DOI: 10.1021/acs.langmuir.5b01897.

\section{AUTHOR INFORMATION}

\section{Corresponding Authors}

*E-mail: hskim@princeton.edu.

*E-mail: hyk@snu.ac.kr.

\section{Notes}

The authors declare no competing financial interest.

\section{ACKNOWLEDGMENTS}

This work was supported by the National Research Foundation (grant nos. 2013034978 and 2014048162) of Korea via SNU IAMD.

\section{REFERENCES}

(1) Stone, H. A.; Stroock, A. D.; Ajdari, A. Engineering flows in small devices: microfluidics toward a lab-on-a-chip,. Annu. Rev. Fluid Mech. 2004, 36, 381.

(2) Kern, W. The evolution of silicon wafer cleaning technology,. J. Electrochem. Soc. 1990, 137 (6), 1887.

(3) Leenaars, A. F. M.; Huethorst, J. A. M.; Van Oekel, J. J. Marangoni drying: a new extremely clean drying process,. Langmuir 1990, 6 (11), 1701.

(4) Plochocki, A. P.; Dagli, S. S.; Andrews, R. D. The interface in binary mixtures of polymers containing a corresponding block copolymer: Effects of industrial mixing processes and of coalescence,. Polym. Eng. Sci. 1990, 30 (12), 741.

(5) Oh, D.-W.; Jin, J. S.; Choi, J. H.; Kim, H.-Y.; Lee, J. S. A microfluidic chaotic mixer using ferrofluid,. J. Micromech. Microeng. 2007, 17 (10), 2077. 
(6) Gaponenko, Y.; Shevtsova, V. Effects of vibrations on dynamics of miscible liquids,. Acta Astronaut. 2010, 66 (1), 174.

(7) Liu, R. H.; stremler, M. A.; Sharp, K. V.; Olsen, M. G.; Santiago, J. G.; Adrian, R. J.; Aref, H.; Beebe, D. J. Passive mixing in a threedimensional serpentine microchannel,. J. Microelectromech. Syst. 2000, 9 (2), 190.

(8) Stroock, A. D.; Dertinger, S. K. W.; Ajdari, A.; Mezić, I.; Stone, H. A.; Whitesides, G. M. Chaotic mixer for microchannels,. Science 2002, 295 (5555), 647.

(9) Suciu, D. G.; Smigelschi, O.; Ruckenstein, E. Some experiments on the Marangoni effect,. AIChE J. 1967, 13 (6), 1120.

(10) Suciu, D. G.; Smigelschi, O.; Ruckenstein, E. The spreading of liquids on liquids,. J. Colloid Interface Sci. 1970, 33 (4), 520.

(11) Lopez, J.; Miller, C. A.; Ruckenstein, E. Spreading kinetics of liquid drops on solids,. J. Colloid Interface Sci. 1976, 56 (3), 460.

(12) Bates, C. M.; Stevens, F.; Langford, S. C.; Dickinson, J. T. Motion and dissolution of drops of sparingly soluble alcohols on water,. Langmuir 2008, 24 (14), 7193.

(13) Svitova, T. F.; Hill, R. M.; Radke, C. J. Clayton J, "Spreading of aqueous trisiloxane surfactant solutions over liquid hydrophobic substrates,. Langmuir 2001, 17 (2), 335.

(14) Tan, E.; Thoroddsen, S. T. Marangoni instability of two liquids mixing at a free surface,. Phys. Fluids 1998, 10 (12), 3038.

(15) Santiago-Rosanne, M.; Vignes-Adler, M.; Velarde, M. G. On the spreading of partially miscible liquids,. J. Colloid Interface Sci. 2001, 234 (2), 375 .

(16) Blanchette, F.; Messio, L.; Bush, J. W. M. The influence of surface tension gradients on drop coalescence,. Phys. Fluids 2009, 21 (7), 072107.

(17) Mohamed-Kassim, Z.; Longmire, E. K. Drop coalescence through a liquid/liquid interface,. Phys. Fluids 2004, 16 (7), 2170.

(18) Chengara, A.; Nikolov, A. D.; Wasan, D. T. Spreading of a water drop triggered by the surface tension gradient created by the localized addition of a surfactant,. Ind. Eng. Chem. Res. 2007, 46 (10), 2987.

(19) Tan, E.; Thoroddsen, S. T. Marangoni instability of two liquids mixing at a free surface,. Phys. Fluids 1998, 10 (12), 3038.

(20) Borcia, R.; Bestehorn, M. Partial coalescence of sessile drops with different miscible liquids,. Langmuir 2013, 29 (14), 4426.

(21) Karpitschka, S.; Hanske, C.; Fery, A.; Riegler, H. Coalescence and noncoalescence of sessile drops: impact of surface forces,. Langmuir 2014, 30 (23), 6826.

(22) Vázquez, G.; Alvarez, E.; Navaza, J. M. Surface tension of alcohol water + water from 20 to $50^{\circ}$ C,. J. Chem. Eng. Data 1995, 40, 611.

(23) Khattab, I. S.; Bandarkar, F.; Fakhree, M. A. A.; Jouyban, A. Density, viscosity and surface tension of water + ethanol mixtures from 293 to 323\&gt;K,. Korean J. Chem. Eng. 2012, 29 (6), 812.

(24) Adrian, R. J.; Westerweel, J. Particle Image Velocimetry; Cambridge University Press: Cambridge, U.K., 2011.

(25) Kim, H.; Westerweel, J.; Elsinga, G. E. Comparison of TomoPIV and 3D-PIV for microfluidic flows,. Meas. Sci. Technol. 2013, 24 (2), 024007.

(26) Poelma, C.; Dickson, W.; Dickinson, M. Time-resolved reconstruction of the full velocity field around a dynamically scaledflapping wing,. Exp. Fluids 2006, 41 (2), 213.

(27) Buckingham, E. On physically similar systems; illustrations of the use of dimensional equations,. Phys. Rev. 1914, 4 (4), 345.

(28) Günther, A.; Jhunjhunwala, M.; Thalmann, M.; Schmidt, M. A.; Jensen, K. F. Micromixing of miscible liquids in segmented gas-liquid flow,. Langmuir 2005, 21 (4), 1547. 